\title{
Absorption of carbon dioxide using ethanolamine-based deep eutectic solvents
}

\section{Zhuo Li, " ${ }^{a}$ Lili Wang, " b Changping Li, ${ }^{* a}$ Yingna Cui, ${ }^{\mathrm{b}}$ Shenmin Li, ${ }^{\mathrm{b}}$ Guang Yang,}

\section{Yongming Shen $* c$}

${ }^{a}$ Research Center for Eco-Environmental Engineering, Dongguan University of Technology, No. 1 University Road Songshanhu Zone, Dongguan City, Guangdong Province, 523106, China ; Tel: 86-769-22861921; E-mail: licpbit@hotmail.com (Changping Li) ; lizhuodl@hotmail.com (Zhuo Li)

${ }^{\mathrm{b}}$ College of Chemical Engineering and the Environment, Dalian University, No. 10 Xuefu Street Development Zone, Dalian City, Liaoning Province, 116622, China; E-mail: wanglili1994@126.com (Lili Wang); cuiyingna@dlu.edu.cn (Yingna Cui); lishenmin@dlu.edu.cn (Shenmin Li); yangguang@dlu.edu.cn (Guang Yang)

${ }^{\mathrm{c}}$ Institute of Environmental and Ecological Engineering, Guangdong University of Technology, No. 100 Waihuan West Road University Town Panyu District, Guangzhou City, Guangdong Province, 510006, China; E-mail: ymshen@dlut.edu.cn (Yongming Shen)

* To whom correspondence should be addressed.

\# Equally contributed to this work.

Pages: 13

Figures: 12 
Supporting information Figure Captions:

Figure S1 ${ }^{1} \mathrm{H}$ NMR spectrum of TMAC/MEA (1:5)

Figure $\mathbf{S 2}{ }^{1} \mathrm{H}$ NMR spectrum of TEAC/MEA (1:5)

Figure S3 ${ }^{1} \mathrm{H}$ NMR spectrum of $\mathrm{ChCl} / \mathrm{MEA}$ (1:5)

Figure S4 ${ }^{1} \mathrm{H}$ NMR spectrum of TMAC/MEA/ LiCl(1:5:0.1)

Figure S5 ${ }^{13} \mathrm{C}$ NMR spectrum of $\mathrm{ChCl} / \mathrm{MEA}(1: 5)$

Figure S6 ${ }^{13} \mathrm{C}$ NMR spectrum of TMAC/MEA/ $\operatorname{LiCl}(1: 5: 0.1)$

Figure S7 Effect of desorption times on desorption residue

Figure S8 Effect of temperature on $\mathrm{CO}_{2}$ absorption

(TMAC/MEA $+10 \% \mathrm{H}_{2} \mathrm{O}$ )

Figure S9 Effect of temperature on $\mathrm{CO}_{2}$ absorption

(TMAC/MEA/MDEA $+10 \% \mathrm{H}_{2} \mathrm{O}$ )

Figure S10 Effect of molar ratio on $\mathrm{CO}_{2}$ absorption

Figure S11 Effect of time on $\mathrm{CO}_{2}$ absorption

Figure S12 Effect of repeated times on $\mathrm{CO}_{2}$ absorption 
Figure S1

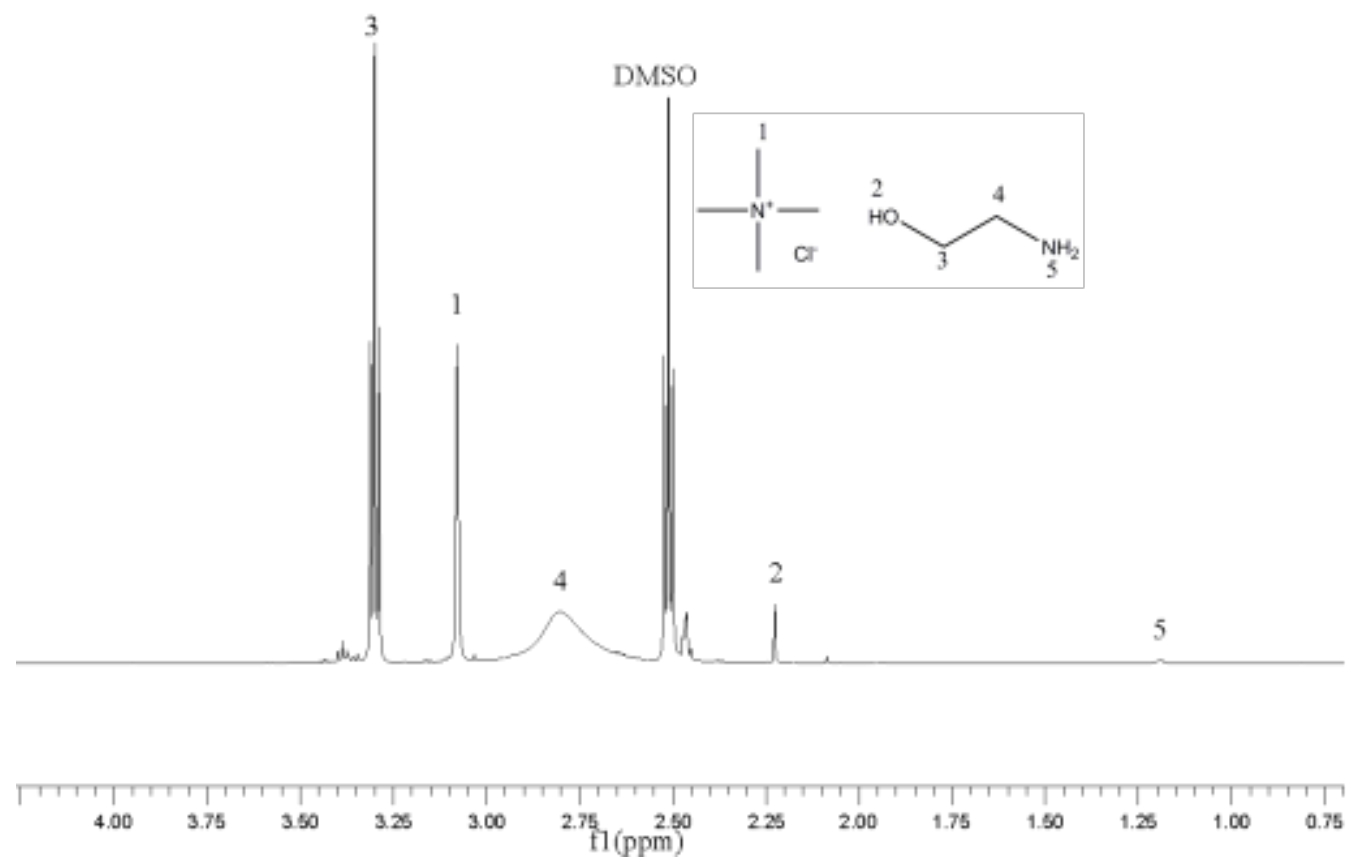


Figure S2

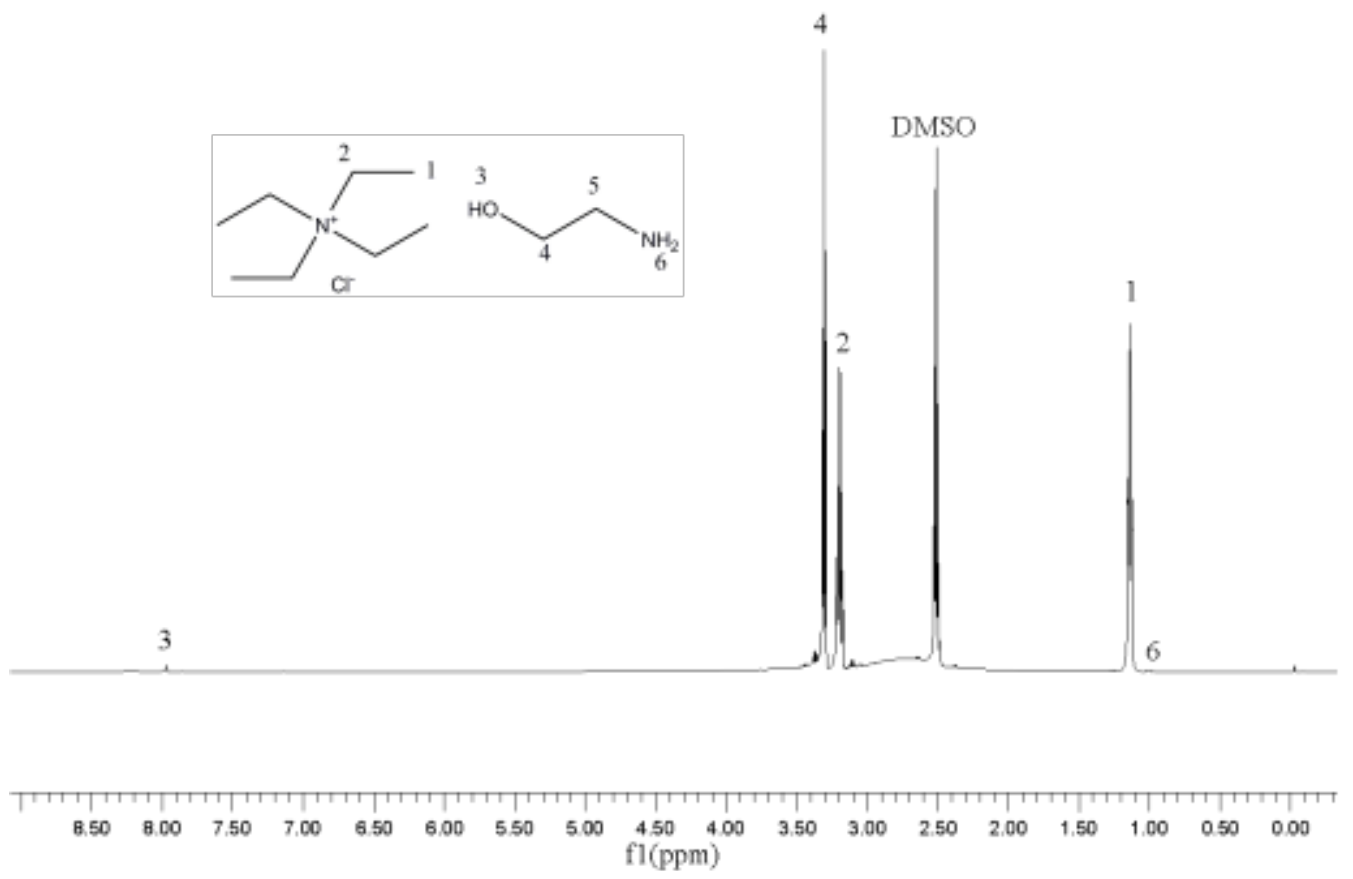


Figure S3

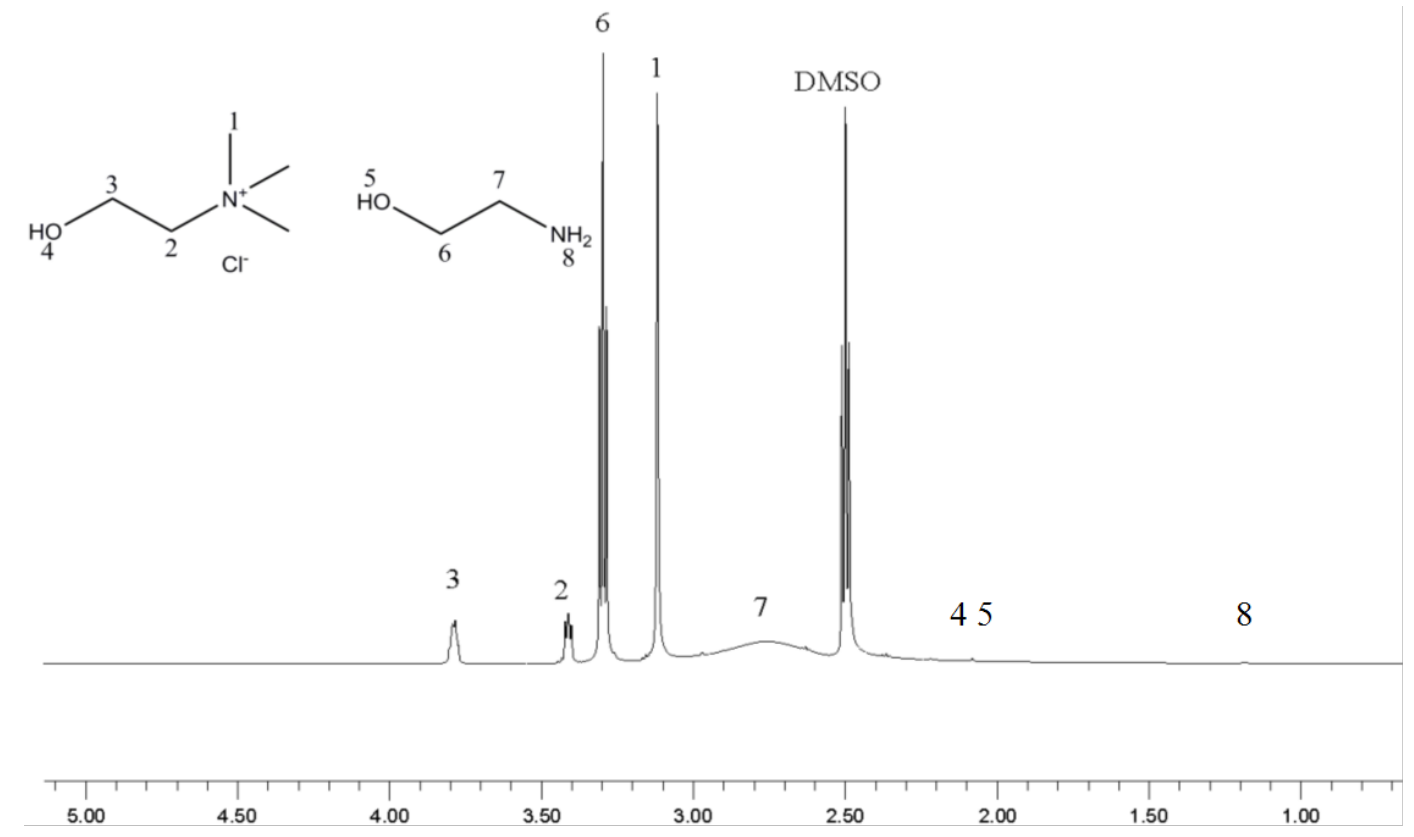


Figure S4

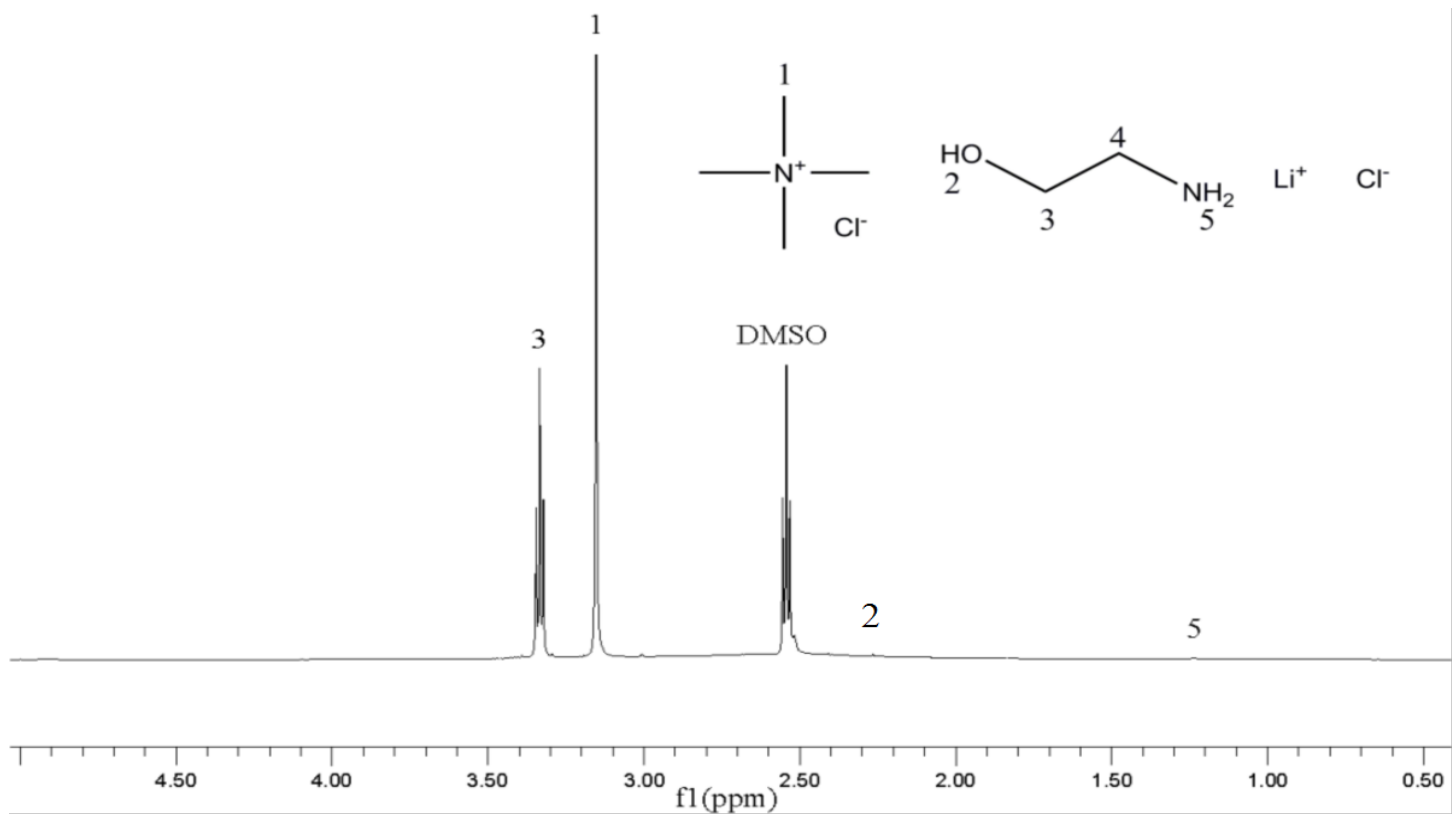

SF 
Figure S5

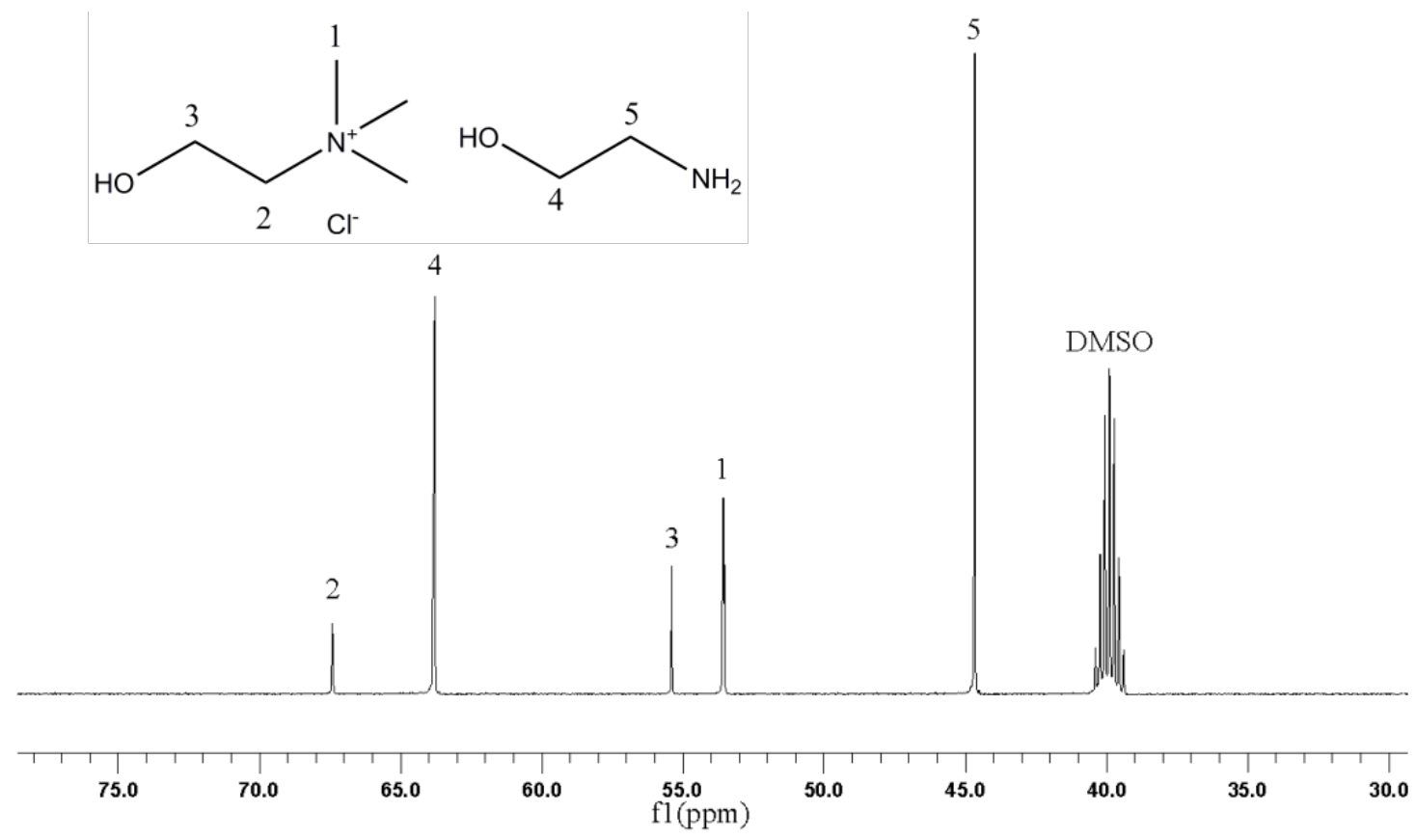


Figure S6

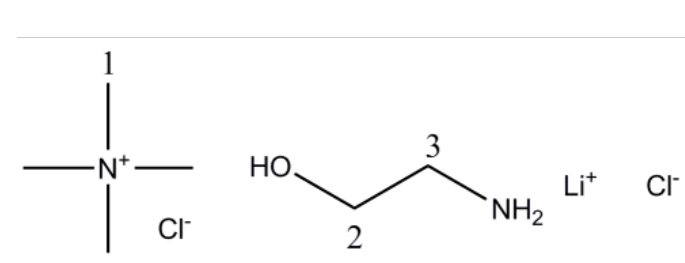

23 DMSO

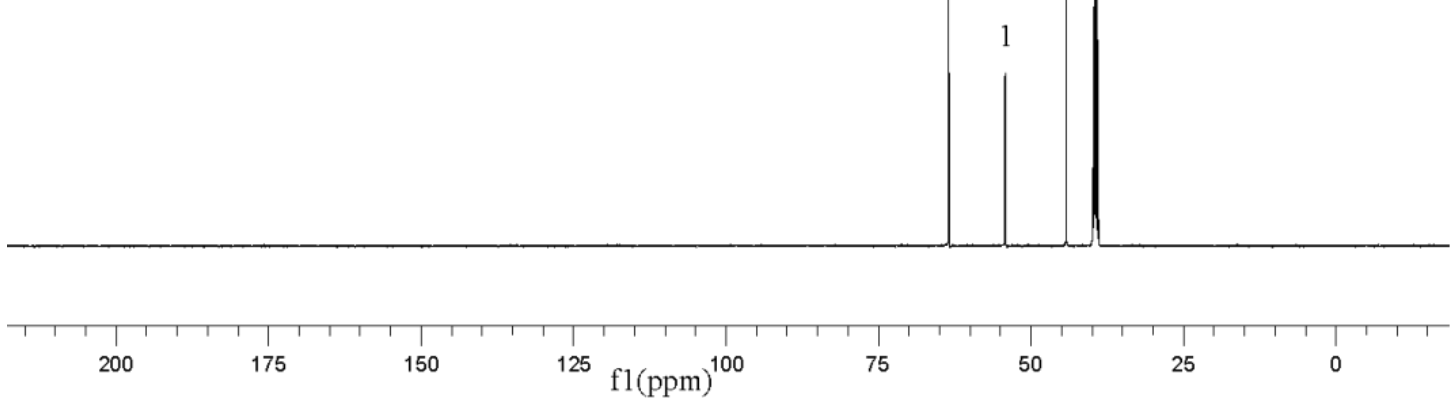


Figure S7

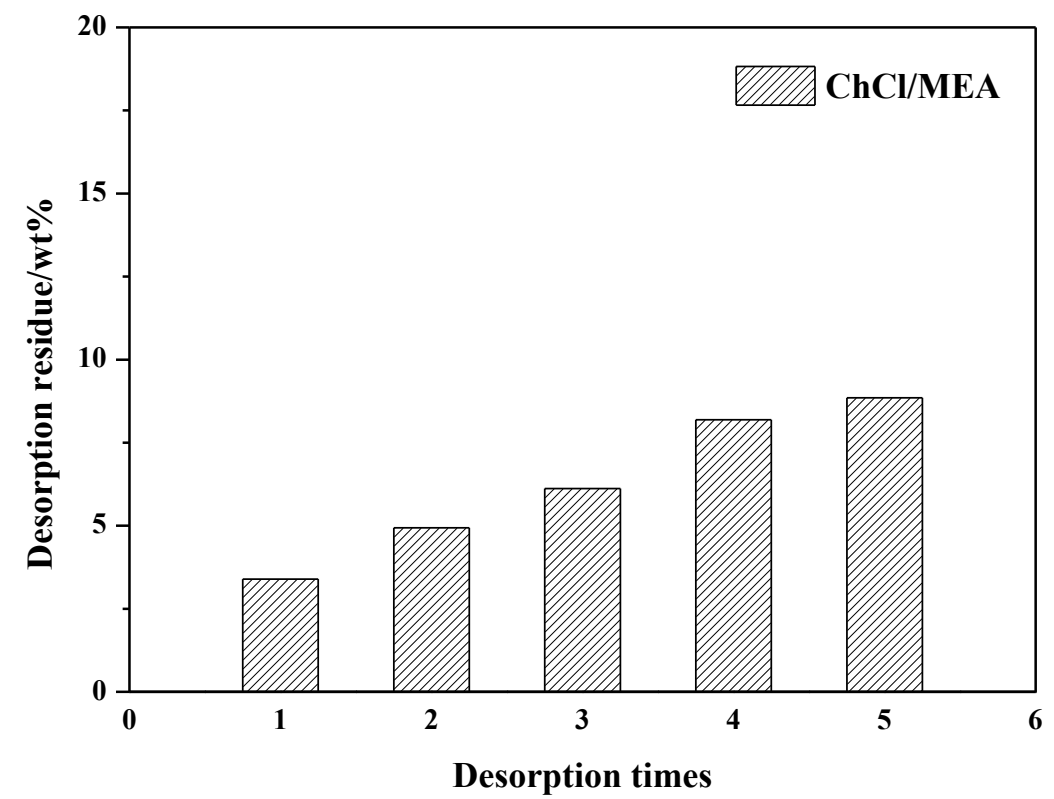


Figure S8

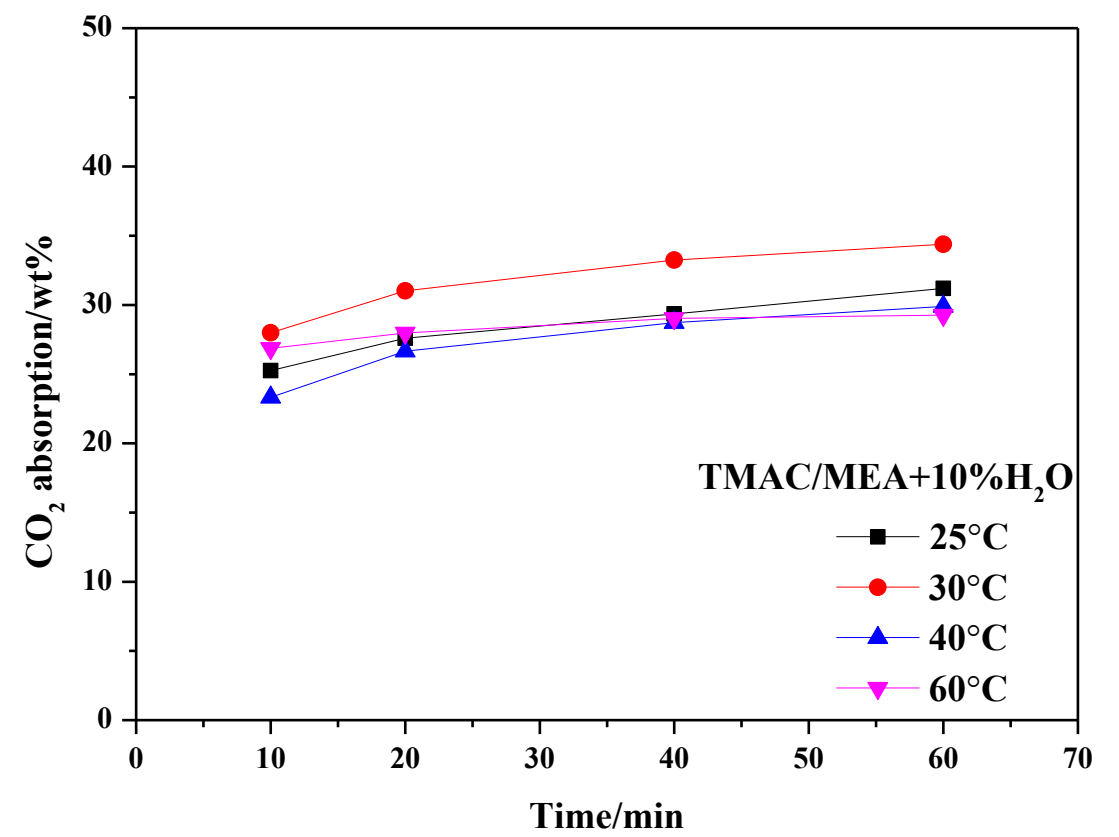


Figure S9

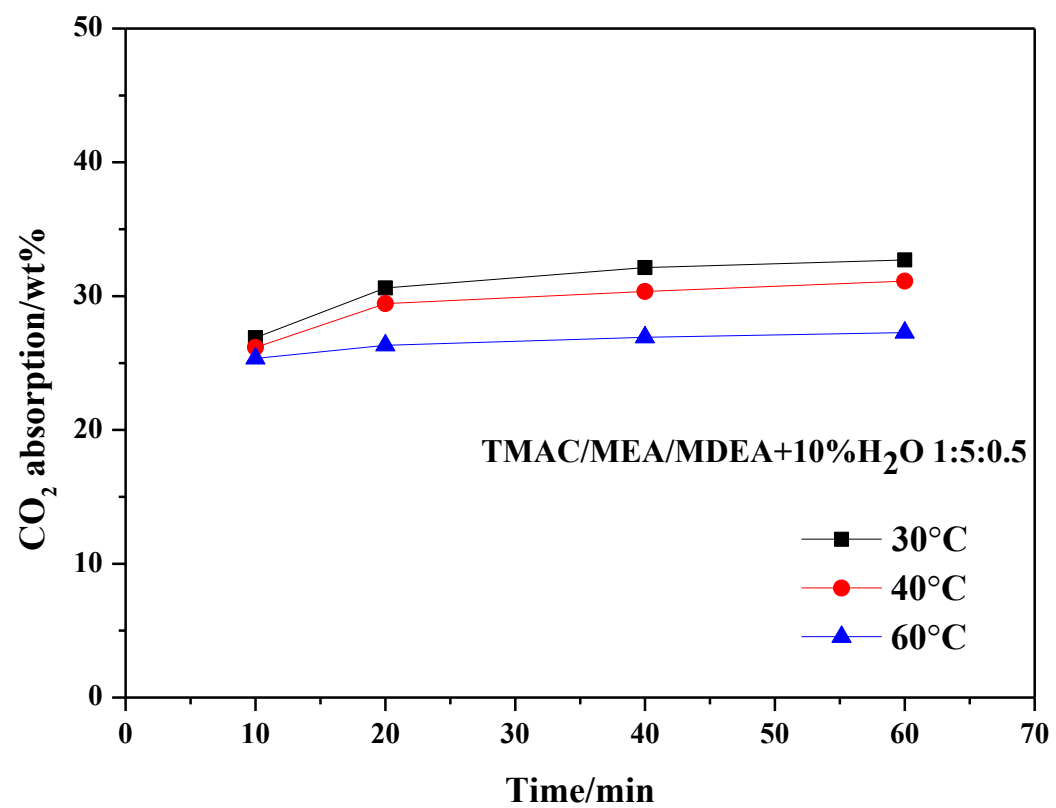


Figure S10

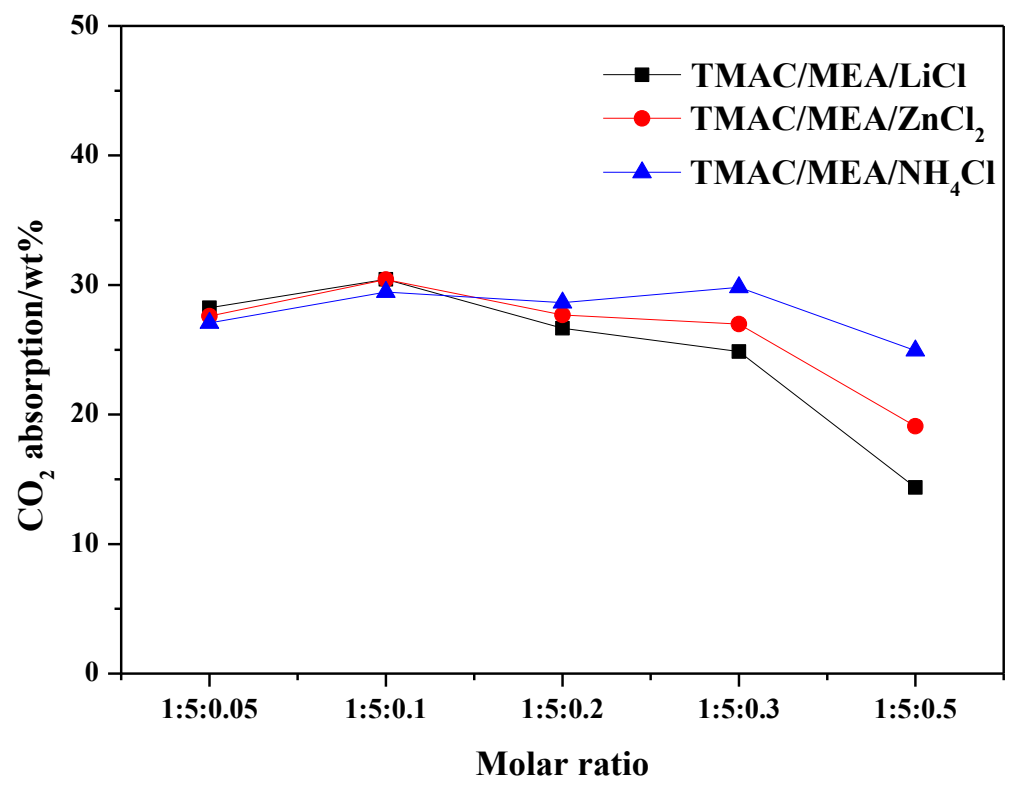


Figure S11

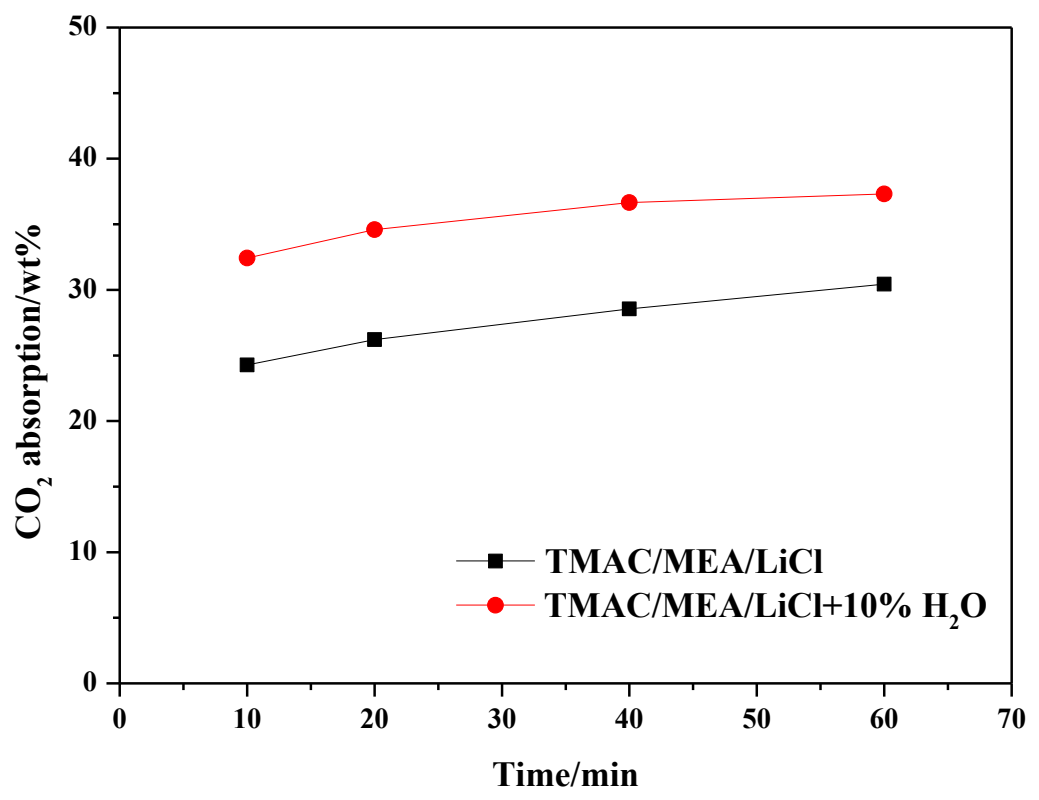


Figure S12

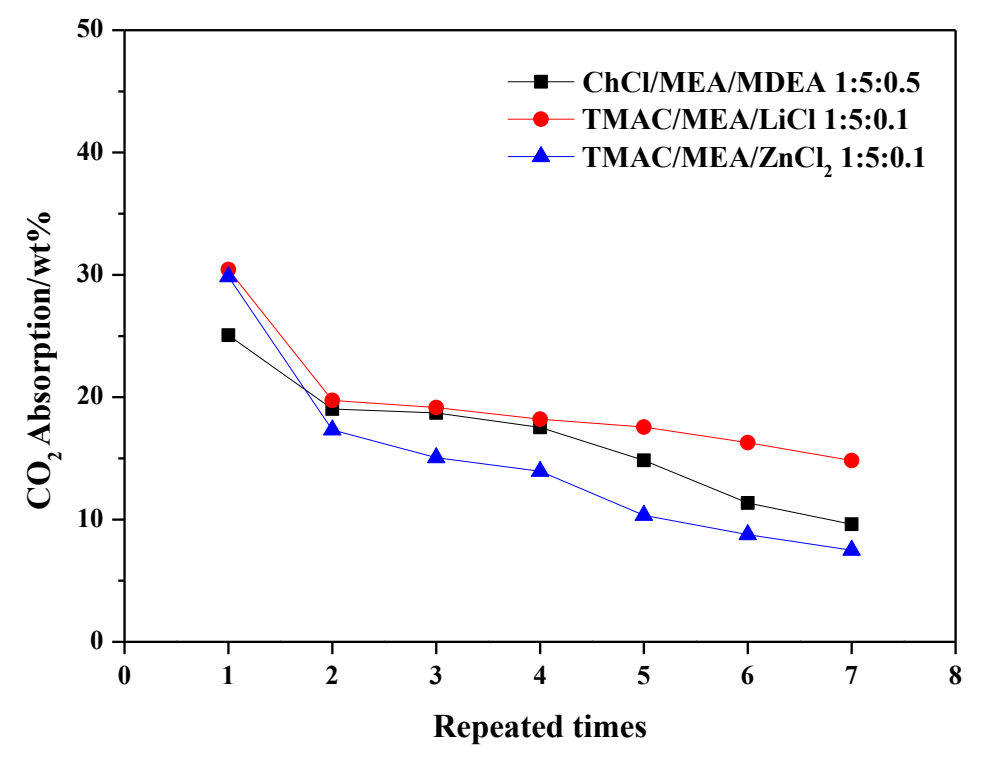

